\title{
SYNTHESIS OF CONFLICT-FREE AIRCRAFT FLIGHT TRAJECTORIES USING MULTI-CRITERIA DYNAMIC PROGRAMMING
}

\author{
Volodymyr KHARCHENKO ${ }^{1}$, Denys VASYLIEV ${ }^{2}$, Volodymyr VASYLIEV ${ }^{3}$
}

National Aviation University, 1 Kosmonavta Komarova Ave., Kyiv 03680, Ukraine



Received 05 October 2015; accepted 17 May 2017
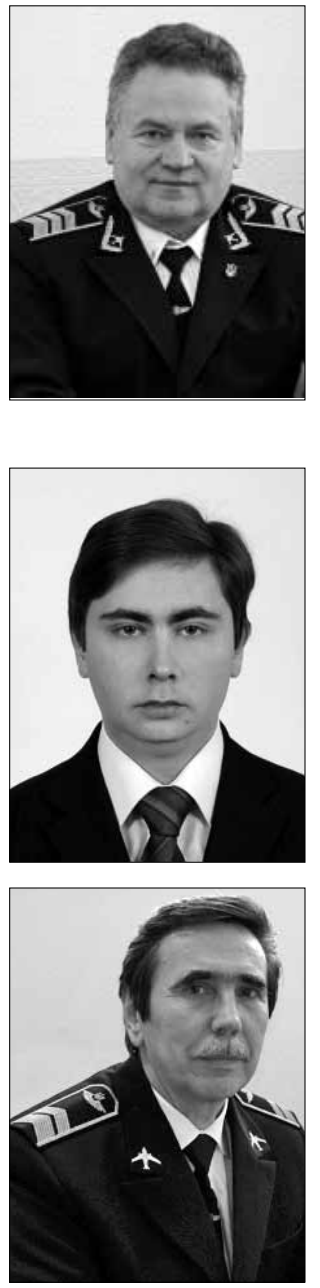

Volodymyr KHARCHENKO, Prof, Dr Sc (Eng)

Date of birth: 15.04.1943.

Education: Kiev Institute of Civil Aviation Engineers, 1967.

Affiliation and functions: engineer at United Aircraft troops (1967-1969), junior and senior researcher (1969-1984), assistant professor (1984-1987), Dr Sci (Eng) (1994), professor (1987-1994) at Kyiv International University of Civil Aviation. Since 2001, he is the vice-rector for scientific-research work at the National Aviation University.

Research interests: aerospace informatics, sociotechnical systems, decision-making under uncertainty and risk.

Publications: author of 477 scientific papers.

Denys VASYLIEV, PhD (Eng)

Date of birth: 06.01.1987.

Education: National Aviation University, Kyiv, 2010.

Affiliation and functions: associate professor at the Department of Air Navigation Systems, National Aviation University, Kyiv, Ukraine.

Research interests: situation analysis and decision-making in air traffic management.

Publications: author of 35 scientific papers.

Volodymyr VASYLIEV, Prof, Dr Sc (Eng)

Date of birth: 08.02.1948.

Education: Kiev Institute of Civil Aviation Engineers, 1972.

Affiliation and functions: head of the Department of Aviation Radio-electronic Complexes, National Aviation University, Kyiv, Ukraine.

Research interests: system theory, identification and prediction, decision making support systems in air navigation and air traffic management.

Publications: author of 125 scientific papers.

Abstract. The paper discusses the problem of flight trajectory synthesis that is conducted to prevent potential conflict situations between two aircraft in air traffic. A method of multi-objective sequential synthesis of conflict-free flight trajectories is developed. This method provides the synthesis of Pareto conflict-free trajectories using multi-criteria dynamic programming and selection of the optimal trajectory. The paper also considers the principles of discretization concerning states and controls to apply dynamic programming. An analysis of the proposed method is performed using computer simulation.

Keywords: aircraft, air traffic management, conflict resolution, decision making, multi-criteria dynamic programming, trajectory synthesis. 


\section{Introduction}

Decision support systems in Air Traffic Management (ATM) are developed to mitigate the level of uncertainty that may arise due to the following factors: inadequate allocation of objects under control in the airspace; delays in data processing, incorrect information about the dynamic characteristics of objects under control, factors associated with low predictability regarding the actions of air traffic controllers and pilots who execute commands, and availability of uncontrollable factors and components affected by the environment.

Conflict resolution is a complex mathematical problem which involves the optimization of trajectories and restriction management. This problem includes conflict detection, clustering, eliminating conflict, and optimization of solutions according to several criteria. A single optimality criterion that characterizes the efficiency of flight is taken into account in many well-known optimization methods when searching for conflict-free aircraft flight trajectories to resolve conflicts (Bicchi, Pallottino 2000; Cafieri et al. 2010; Cetek 2009; Frazzoli et al. 2001; Hu et al. 2000; Richards, How 2002; Tomlin et al. 1998). Considering a single optimality criterion does not allow finding the most effective solution in general. Besides, it should be noted that the methods mentioned above are not used in air traffic control (ATC) nowadays.

EUROCONTROL (European Organization for the Safety of Air Navigation) and the National Aerospace Laboratory of the Netherlands are developing decisionmaking support systems to be used for identifying and resolving conflicts. The EUROCONTROL Conflict Resolution Assistant (CORA) project aims to produce a controller-centered approach to conflict resolution (EUROCONTROL 2003; Kirwan, Flynn 2001). Today, the development of the system prototype is known as CORA-2, which helps to automatically identify possible conflict-free aircraft flight trajectories and to rank them in accordance with twelve optimality criteria. The advantage of CORA-2 is its easy integration into air traffic control (ATC) automated systems. Nevertheless, CORA2 has several significant disadvantages. Firstly, the construction of conflict-free trajectories does not consider combined maneuvers to change the direction, speed and altitude, which significantly narrows the possibility to effectively resolve a conflict. Secondly, the application of a brute-force method for constructing a set of possible maneuver trajectories reduces the computational efficiency of the algorithm. Thirdly, the flight efficiency criterion is not considered when assessing the effectiveness of possible conflict-free trajectories, whereas other criteria, which essentially overlap, are taken into account. Finally, it is not determined how to establish the values of optimal criteria coefficients.
Algorithms based on force field methods (Eby 1994; Eby, Kelly 1999; Kosecka et al. 1997; Zeghal 1998) synthesize conflict-free trajectories which are complicated or even unrealizable for real aircraft.

The common disadvantage of the methods considered is that they do not provide the synthesis of maneuvers using a combination of heading, speed and altitude changes.

\section{Problem statement}

A conflict situation is understood as the predicted approach of two aircraft towards each other in airspace and time when the separation standards are violated. Conflict resolution is considered to be a multi-criteria problem of decision-making, which is to select conflict-free flight trajectories taking into account several optimality criteria and constraints. An alternative choice of aircraft flight trajectories is possible, selecting trajectories using which an aircraft maneuvers to avoid a conflict. Maneuvering is understood as the change in direction and flight speed. An absolute constraint is flight safety maintained by the separation standards.

The optimality criteria characterizing the process of conflict avoidance (prevention) are flight regularity $c_{1}$, flight efficiency $c_{2}$, and complexity of maneuvering $c_{3}$ $c_{3}$. The indicators of these criteria are: deviation from a flight plan, fuel consumption, and the number of flight profile changes. Based on the selected optimality criteria, a vector is created, as seen in the following expression:

$$
\mathbf{C}=\left\{c_{i}\right\}, i=\overline{1,3} \text {. }
$$

To resolve a conflict is to select flight trajectory $\mathbf{T}^{*}$ which ensures the elimination of the conflict and complies with the flight regularity criterion (minimal deviations from a flight plan), the flight efficiency criterion (minimal fuel consumption), and the criterion of the complexity of maneuvering (minimal number of flight profile changes):

$$
\mathbf{T}^{*}=\arg \min _{\mathbf{T} \in \Omega} \mathbf{C}(\mathbf{T}),
$$

where $\Omega$ indicates the set of possible conflict-free flight trajectories.

\section{The method of multi-criteria sequential synthesis of conflict-free flight trajectories}

3.1. The synthesis of the set of Pareto-efficient conflict-free flight trajectories using multi-criteria dynamic programming

Preventing potential conflicts is considered to be a sequential multistage process of decision-making at discrete points in time. The objective of the conflict-free trajectory synthesis is to find a route for the aircraft, that would eliminate a conflict situation and minimize deviations from a flight plan, fuel consumption, and a number of flight profile changes when transferring it 
from the initial state (conflict detected) to the final state (exiting the ATC area).

Conflict resolution is a controllable process, and an aircraft performing maneuvers is defined as a discrete dynamic system, $\mathbf{S}$. Thus, the conflict-free flight trajectory synthesis is intended to optimally control the dynamic system $\mathbf{S}$ by using the method of dynamic programming. The use of the multi-criteria dynamic programming method allows synthesizing the set of Pareto-efficient conflict-free flight trajectories, $\mathbf{P}$. The next optimal trajectory, $\mathbf{T}^{*}$, can be selected from this set.

The trajectory synthesis is observed in time interval $\left[t_{0}, t_{k}\right]$, where $t_{0}$ is the moment that a potential conflict is detected, and $t_{k}$ is the time during which an aircraft is within an ATC area. The discretization of dynamic system $S$ is carried out depending on time. Dynamic system $\mathbf{S}$ is determined as the expression consisting of the following indices:

$$
\mathbf{S}=\left\{\mathbf{D}_{\mathbf{X}}, \mathbf{X}_{0}, \mathbf{X}_{k}, \mathbf{D}_{\mathbf{U}}(\mathbf{X}), \mathbf{D}_{\mathbf{U}}^{S}(\mathbf{X}), f(\mathbf{X}, \mathbf{U}), \Delta J_{i}(\mathbf{X}, \mathbf{U}), \mathbf{T}_{r e f}\right\},
$$

where $\mathbf{D}_{\mathrm{X}}$ is the set of conflict-free states of the aircraft which performs the maneuvers; $\mathbf{X}_{0}, \mathbf{X}_{k}$ - the initial and the final state of the aircraft which performs the maneuvering; $\mathbf{D}_{\mathbf{U}}(\mathbf{X})$ - the set of possible controls $\mathbf{U}$ in state $\mathbf{X}$; $\mathbf{D}_{\mathbf{U}}^{S}(\mathbf{X})$ - the set of conflict-free controls $\mathbf{U}$ in state $\mathbf{X}$; $f(\mathbf{X}, \mathbf{U})$ - the transit function from state $\mathbf{X}$ under the action of control $\mathbf{U} ; \Delta J_{i}(\mathbf{X}, \mathbf{U})$ - the exes after each $i$ optimality criteria when transiting from state $\mathbf{X}$ under the action of control $\mathbf{U}, i=\overline{1,3} ; \mathbf{T}_{\text {ref }}$ - the discretized planned trajectory of an aircraft that does not maneuver.

The vector of state $\mathbf{X}$ contains the coordinates, flight speed, and heading. The initial $\mathbf{X}_{0}$ and final $\mathbf{X}_{k}$ states of the system are conflict-free. The vector of entering control signals $\mathbf{U}$ contains specific values of the flight speed and bank angle. The possible controls are limited in accordance with aircraft flight performances and the requirements pertaining to the passenger comfort while maneuvering. These constraints determine the set of possible controls $\mathbf{D}_{\mathbf{U}}(\mathbf{X})$ in state $\mathbf{X}$. In general, it is considered that the aircraft may transit into state $\mathbf{X}(j)$ at stage $j$ from several states $\mathbf{X}(j-1)$ at the previous stage $(j-1)$ :

$$
\mathbf{X}(j)=f(\mathbf{X}(j-1), \mathbf{U}(j-1)) .
$$

The final state $\mathbf{X}_{k}$ is specified only by the horizontal coordinates of the point at which an aircraft is within an ATC area. It is expected that an aircraft may transit into the final state from all states of the previous stage. The process of dynamic programming combines the consistent determination of conflict-free states and the relevant Pareto-effective controls.

Time interval $\left[t_{j-1}, t_{j}\right], t_{j}=t_{j-1}+\Delta t$ corresponds to each stage $j$, except for the last one, where $\Delta t$ is the discretization step. In general, the time interval of the last $k$ stage is different for all controls, because of the different time of reaching the fixed final state when transiting from the states at the previous stage $(k-1)$.

At each $j$ stage of conflict resolution, the following factors are determined:

- sets of possible controls $\mathbf{D}_{\mathbf{U}}(\mathbf{X}(j-1))$ in the states of $(j-1)$ stage;

- the violation of the separation standards when transiting from states $\mathbf{X}(j-1)$ at $(j-1)$ stage under the action of controls $\mathbf{U}(j-1) \in \mathbf{D}_{\mathbf{U}}(\mathbf{X}(j-1))$, and the corresponding sets of conflict-free controls $\mathbf{D}_{\mathbf{U}}^{S}(\mathbf{X}(j-1)) \in \mathbf{D}_{\mathbf{U}}(\mathbf{X}(j-1))$;

- simulated aircraft flight trajectories, the set of conflict-free states $\mathbf{D}_{\mathbf{X}}(j)$ and expenses $\Delta J_{i}(\mathbf{X}(j-1), \mathbf{U}(j-1))$ when transiting from states $\mathbf{X}(j-1)$ at $(j-1)$ stage under the action of conflict-free controls $\mathbf{U}(j-1) \in \mathbf{D}_{\mathbf{U}}^{S}(\mathbf{X}(j-1))$;

- sets of Pareto-effective evaluations of conflict-free trajectories $\mathbf{E}(\mathbf{X}(j))$ when transiting into states $\mathbf{X}(j) \in \mathbf{D}_{\mathbf{X}}(j)$.

To predict the violations of the separation standards between aircraft, different methods may be used. When applying the geometric method, it is possible to determine horizontal distance $d_{\min }\left(\mathbf{X}(j-1), \mathbf{U}(j-1), \mathbf{T}_{r e f}\right)$ and vertical interval $\Delta h_{\min }\left(\mathbf{X}(j-1), \mathbf{U}(j-1), \mathbf{T}_{r e f}\right)$ between aircraft at the point of the closest approach within a time interval $\left[t_{j-1}, t_{j}\right]$. In case the separation standards are violated, control $\mathbf{U}(j-1)$ is considered to be a conflict one: $\left(d_{\text {min }}<d_{S}\right) \wedge\left(\Delta h_{\text {min }}<h_{S}\right) \Rightarrow \mathbf{U}(j-1) \notin \mathbf{D}_{\mathbf{U}}^{S}(\mathbf{X}(j-1))$.

In order to provide a safe separation of aircraft in terms of probability, it is possible to use methods of estimating the conflict situation probability or methods of estimating the risk of aircraft collision (Babak et al. 2007). Using the above mentioned methods, it is possible to determine the probability $P\left(\mathbf{X}(j-1), \mathbf{U}(j-1), \mathbf{T}_{r e f}\right)$ of the violation of the separation standards between aircraft within time interval $\left[t_{j-1}, t_{j}\right]$. In the case that this probability exceeds the threshold value of $P_{S}$, control $\mathbf{U}(j-1)$ is considered to be a conflict one:

$$
P>P_{S} \Rightarrow \mathbf{U}(j-1) \notin \mathbf{D}_{\mathbf{U}}^{S}(\mathbf{X}(j-1)) \text {. }
$$

When deviations from the flight plan, fuel consumption and the number of flight profile changes are used as efficiency indices $J$, it is possible to provide the additivity of expenses $\Delta J(\mathbf{X}, \mathbf{U})$ when transiting from one state into another. For an arbitrary flight trajectory $\mathbf{T}=\left\{\mathbf{X}_{0}, \mathbf{X}(1), \ldots \mathbf{X}(m)\right\}$, the value of indices $J$, which characterizes flight efficiency is determined as follows:

$$
J_{i}(\mathbf{T})=\sum_{j=1}^{m} \Delta J_{i}(\mathbf{X}(j-1), \mathbf{U}(j-1)) .
$$


Indices $J$ provide vector $\mathbf{J}=\left\{J_{i}\right\}$. To solve the problem of the synthesis of Pareto-effective conflict-free trajectories, a direct procedure of multi-criteria dynamic programming is used. To determine the set of Pareto-effective evaluations of conflict-free trajectories $\mathbf{E}(\mathbf{X}(j))$ when transiting into state $\mathbf{X}(j) \in \mathbf{D}_{\mathbf{X}}(j)$ at $j$ stage from state $\mathbf{X}(j-1) \in \mathbf{D}_{\mathbf{X}}(j-1)$ at the previous stage $(j-1)$, the equation of multi-criteria dynamic programming is used (Vasyliev 2014; Klamroth, Wiecek 2000):

$$
\begin{aligned}
& \mathbf{E}(\mathbf{X}(j))= \\
& \text { eff } \bigcup_{\mathbf{X}(j-1) \in \oplus}\left(\mathbf{E}(\mathbf{X}(j-1)) \oplus\left\{\Delta J_{i}\left(\mathbf{X}(j-1), \mathbf{U}^{\prime}(j-1)\right)\right\}\right),(7)
\end{aligned}
$$

where eff is the mathematical operator for the determination of Pareto-effective evaluations; $\oplus$ - a direct sum; $\Pi$ - a set of states at stage $(j-1)$, due to which the transition into state $\mathbf{X}(j)$ is possible; $\mathbf{U}^{\prime}(j-1) \in \mathbf{D}_{\mathbf{U}}^{S}(\mathbf{X}(j-1))$ are the controls which allow an aircraft to transit from state $\mathbf{X}(j-1) \in \boxplus$ into state $\mathbf{X}(j)$.

In equation (8), value $\mathrm{K}$ is a set of full flight trajectories by which an aircraft transits from the initial state $\mathrm{X}_{0}$ into the final state $\mathrm{X}_{k}$. As a result, taking into account equation (7), the set of Pareto-effective conflict-free flight trajectories $\mathrm{P}$ is determined as follows:

$$
\mathbf{P}=\left\{\mathbf{T} \in \mathbf{K} \mid \mathbf{J}(\mathbf{T}) \in \mathbf{E}\left(\mathbf{X}_{k}\right)\right\} .
$$

\subsection{Discretization of states and controls}

Generally, the use of discrete dynamic programming requires the ability of aircraft to transit into state $\mathbf{X}(j) \in \mathbf{D}_{\mathbf{X}}(j)$ at $j$ stage from several states $\mathbf{X}(j-1) \in \mathbf{D}_{\mathbf{X}}(j-1)$ at the previous stage $(j-1)$. The integration of fixed states and controls, which allow to transit from several states $\mathbf{X}(j-1)$ at the previous stage to one state $\mathbf{X}(j)$, is a difficult problem. Conflict-free states are the outcome of the flight trajectory synthesis. In this case, the integration of fixed states is impossible. Therefore, it is proposed to combine the sequential determination of conflict-free states and the relative Pareto-effective controls using interpolation when solving the problem of dynamic programming.

It is considered that maneuvering aircraft may change direction and flight speed at all stages except the last one. Thus, being in the defined state $\mathbf{X}(j-1) \in \mathbf{D}_{\mathbf{X}}(j-1), j=\overline{1, k-1}$, an aircraft may maintain the flight direction and perform a left or right turn with bank $\gamma$, maintaining the flight speed and increasing or decreasing it by value $\Delta V$. Consequently, the basic set of controls $\mathbf{D}_{\mathbf{U} 0}(\mathbf{X}(j-1))$ includes 9 possible combinations of control. The set of possible controls $\mathbf{D}_{\mathbf{U}}(\mathbf{X}(j-1))$ is a subset of the set described as $\mathbf{D}_{\mathbf{U} 0}(\mathbf{X}(j-1))$. This leads us to conclude that the set of conflict-free controls $\mathbf{D}_{\mathbf{U}}^{S}(\mathbf{X}(j-1))$ is a subset of the set described as
$\mathbf{D}_{\mathbf{U} 0}(\mathbf{X}(j-1))$ as well. When using controls from the set described as $\mathbf{D}_{\mathbf{U}}^{S}(\mathbf{X}(j-1)) \in \mathbf{D}_{\mathbf{U} 0}(\mathbf{X}(j-1))$, an aircraft transits into different states at $j$ stage:

$$
\begin{aligned}
& \mathbf{X}^{\prime}(j)=f(\mathbf{X}(j-1), \mathbf{U}(j-1)), \mathbf{X}^{\prime}(j) \in \mathbf{D}_{\mathbf{X}}(j), \\
& \mathbf{U}(j-1) \in \mathbf{D}_{\mathbf{U}}^{S}(\mathbf{X}(j-1)),
\end{aligned}
$$

It is proposed to implement a rule for the formation of new states $\mathbf{X}(j)$ which combine states $\mathbf{X}^{\prime}(j)$. The proximity of the location and direction as well as the equality of flight speeds are the background for combining states. As a result, it is considered that an aircraft may transit into state $\mathbf{X}(j)$ under the action of several controls $\mathbf{U}^{\prime}(j-1)$. Under the action of these controls, an aircraft transits into states $\mathbf{X}^{\prime}(j)$, which are combined to obtain state $\mathbf{X}(j)$. The set described as $\Pi$ is different for each state described as $\mathbf{X}(j) \in \mathbf{D}_{\mathbf{X}}(j)$.

The coordinates and flight route of an aircraft in the new state $\mathbf{X}(j)$ are determined as the arithmetic mean of these parameters for the states of $\mathbf{X}^{\prime}(j)$, which are combined in this new state. Expense value $\Delta J_{i}\left(\mathbf{X}(j-1), \mathbf{U}^{\prime}(j-1)\right)$ when transiting into the new states $\mathbf{X}(j)$ from the states of set $\Pi$ is determined using the nearest-neighbor interpolation of expenses $\Delta J_{i}^{\prime}(\mathbf{X}(j-1), \mathbf{U}(j-1))$ for states $\mathbf{X}^{\prime}(j)$, which are combined. At the last stage, an aircraft performs its flight by transiting into state $\mathbf{X}_{k}$ from all states at the previous stage with no change in the flight speed. The value of bank angle $\gamma$ is determined by a special control law.

\subsection{The optimal trajectory selection from the Pareto set}

The selection of the optimal conflict-free aircraft flight trajectory $\mathbf{T}^{*}$ is performed by means of narrowing the set of Pareto-effective trajectories $\mathbf{P}$ using the method of convolution of vector optimality criterion $\mathbf{C}$. It is proposed to select the optimal trajectory $\mathbf{T}^{*}$ according to the following equation:

$$
\mathbf{T}^{*}=\arg \min _{\mathbf{T} \in \mathbf{P}} \max _{\mathbf{W} \in \mathbf{D}_{w}} \sum_{i=1}^{3} w_{i} c_{i}(\mathbf{T}),
$$

where $c_{i}$ is the optimality criteria with the range of allowable values $\mathbf{D}_{c}=\{c \mid c \in[0,1]\} ; w_{i}$ - coefficients which display the relative significance of the criteria and form vector $\mathbf{W}=\left\{w_{i}\right\}, i=\overline{1,3}$ with the range of allowable values $\mathbf{D}_{w}, \max _{\mathbf{W} \in \mathbf{D}_{w}} \sum_{i=1}^{3} w_{i} c_{i}(\mathbf{T})$ - the problem of linear programming.

Equation (10) corresponds to a discreet strategy of decision-making, when the choice of the optimal trajectory comes to the selection of the best trajectory from the worst ones. The values of the optimality criteria of trajectory $\mathbf{T}$ of set $\mathbf{P}$ are applied to the range of allowable values $\mathbf{D}_{c}$, with the help of a positive linear transformation:

$$
c_{i}(\mathbf{T})=\frac{J_{i}(\mathbf{T})-\min _{\mathbf{T} \in \mathbf{P}} J_{i}(\mathbf{T})}{\max _{\mathbf{T} \in \mathbf{P}} J_{i}(\mathbf{T})-\min _{\mathbf{T} \in \mathbf{P}} J_{i}(\mathbf{T})},
$$

where $J_{i}(\mathbf{T})$ indicates the value of the index which determines a particular optimality criterion. 
The range of allowable values of coefficients $\mathbf{D}_{w}$ is determined taking into account that the values of the selected optimality criteria are ranked in a decreasing order, and the coefficients cannot be less than the specified parameter $w_{0}>0$ :

$$
\begin{gathered}
c_{1} \succ c_{2} \succ c_{3} \Leftrightarrow w_{1} \geq w_{2} \geq w_{3}, \\
\mathbf{D}_{w}=\left\{\mathbf{W} \mid \sum_{i=1}^{3} w_{i}=1 ; w_{i} \geq w_{i+1}, i=\overline{1,2} ; w_{3} \geq w_{0}>0\right\} .
\end{gathered}
$$

\section{Computer simulation}

\subsection{Input data}

The proposed method of the multi-criteria sequential synthesis of conflict-free flight trajectories is analyzed using computer simulation. The researchers simulate a conflict situation which occurs between two aircraft with intersecting trajectories performing flights with a constant speed at the same flight level. The value of the horizontal separation is specified as $d_{s}=20 \mathrm{~km}$. The geometric method is applied to predict the violation of the separation standards.

The initial parameters of aircraft flight and the characteristics of the predicted conflict situation are shown in Table 1. It is presumed that Aircraft 1 performs maneuvering to avoid the conflict. Aircraft 2 uses the planned trajectory.

Table 1. Parameters of aircraft flight and the characteristics of the predicted conflict situation

\begin{tabular}{lcc}
\hline \multicolumn{1}{c}{ Parameters } & Aircraft 1 & Aircraft 2 \\
\hline Heading $\varphi$, degrees & 0 & 80 \\
\hline Cruising speed $V, \mathrm{M} / \mathrm{s}$ & 220 & 210 \\
\hline Initial coordinates $\left(x_{0} ; y_{0}\right), \mathrm{km}$ & $(65 ; 0)$ & $(0 ; 40)$ \\
\hline Distance to the check point $L_{0}, \mathrm{~km}$ & 120 & - \\
\hline $\begin{array}{l}\text { Planned time of flying over the } \\
\text { check point } t_{k}, \mathrm{~s}\end{array}$ & 545 & - \\
\hline $\begin{array}{l}\text { Estimated time of flying to the } \\
\text { closest point of approach } t_{\text {min } 0}, \mathrm{~s}\end{array}$ & \multicolumn{2}{c}{272} \\
\hline $\begin{array}{l}\text { Estimated minimum distance } \\
\text { between aircraft } d_{\text {min } 0}, \mathrm{~m}\end{array}$ & \multicolumn{2}{c}{13227} \\
\hline
\end{tabular}

It is considered that Aircraft 1 changes the flight direction and speed to avoid the conflict. The bank equals $\gamma=20^{\circ}$ on the turns. The time of the turn is limited to fifteen seconds. The value of the flight speed change is equal to $\Delta V=5 \mathrm{~m} / \mathrm{s}$. The lateral deviation from the planned flight trajectory is limited to $15 \mathrm{~km}$. The simulation of flight trajectories is carried out taking into account the dynamics of controllable aircraft movement, aircraft performance filed in the EUROCONTROL Base of Aircraft Data (BADA), and fuel consumption. A Boeing 737-800 aircraft is selected. The synthesis of conflict-free trajectories is discretized for 7 stages. The discretization step for the stages from 1 to 6 is equal to $\Delta t=60 \mathrm{~s}$.

\subsection{Results of the simulation}

As a result of the simulation, the following is determined:

- a set of Pareto-effective conflict-free trajectories $\mathbf{P}$, which contains 21 trajectories;

- the values of the optimality criteria for the trajectories from set $\mathbf{P}$;

- an optimal conflict-free flight trajectory for Aircraft 1 (Table 2).

Figure 1 shows the Pareto-effective conflict-free trajectories and the planned trajectory of Aircraft 1 in the space-time coordinate system $x \times y \times t$.



Fig. 1. The set of Pareto-effective flight trajectories of Aircraft 1 in the space-time coordinate system: 1 - planned trajectory; 2 - planned trajectory in the airspace coordinate system; 3 check point on the route; 4 - Pareto-effective conflict free trajectories; 5 - states at different stages

The set of Pareto-effective trajectories $\mathbf{P}$ is characterized by the following indices: the minimum and maximum absolute deviations from the planned flight time that equal $\Delta t_{k \min }=0.3$ and $\Delta t_{k \max }=27.8$ seconds respectively; the minimum and maximum fuel consumption increase, as compared with the planned flight trajectory, $\Delta q_{\min }=0.3 \%$ and $\Delta q_{\max }=3 \%$; the maximum and minimum number of flight profile changes, $n_{\min }=3, n_{\max }=11$. The optimality criteria values for the Pareto-effective trajectories are shown in Figure 2.

The value of the objective function: $\max _{\mathbf{W} \in \mathbf{D}_{w}} \sum_{i=1}^{3} w_{i} c_{i}(\mathbf{T})$ with $w_{0}=0.1$, is shown in Figure 3 .

The parameters of the conflict-free optimal flight trajectory of Aircraft 1 are shown in Table 2. Figure 4 shows the conflict-free flight trajectory. The specified flight speeds are given in Table 3. 


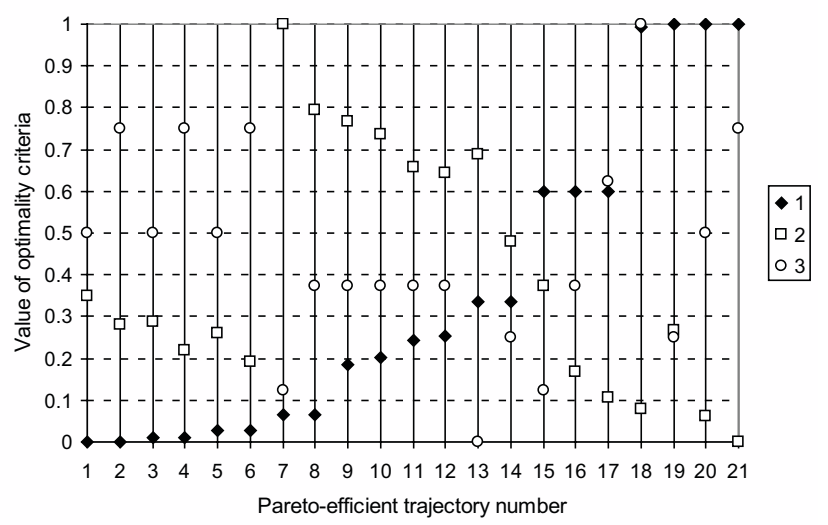

Fig. 2. Optimality criteria values: $1-$ regularity criterion $c_{1} ; 2-$ efficiency criterion $c_{2} ; 3$ - criterion of maneuvering complexity $c_{3}$

Table 2. Parameters of the conflict-free optimal flight trajectory

\begin{tabular}{ll}
\hline \multicolumn{1}{c}{ Parameters } & \multicolumn{1}{c}{ Values } \\
\hline Pareto-effective trajectory № & 5 \\
\hline Minimum distance between two aircraft $d_{\text {min }}, \mathrm{m}$ & 20364 \\
\hline Deviation from the planned flight time $\Delta t_{k}, \mathrm{~s}$ & 1,1 \\
\hline Additional fuel consumption $\Delta q, \%$ & 1 \\
\hline Number of profile changes $n$ & 7 \\
\hline
\end{tabular}

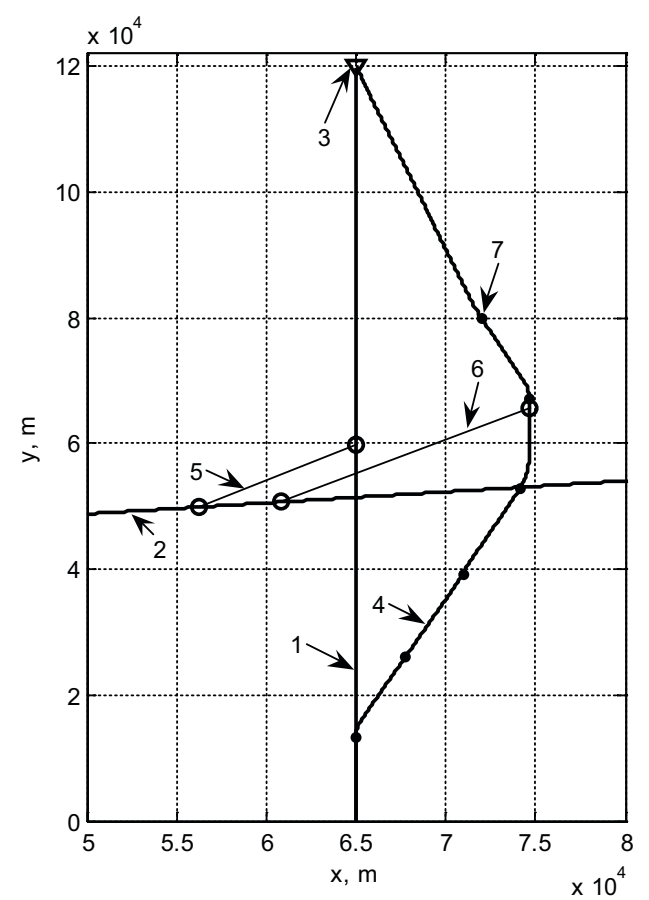

Fig. 4. Aircraft flight trajectories when resolving the conflict situation: 1 - planned flight trajectory of Aircraft $1 ; 2$ - planned flight trajectory of Aircraft 2;3 - check point; 4 - optimal conflict-free flight trajectory of aircraft $1 ; 5$ - the minimum distance between two aircraft when detecting the conflict; 6 the minimum distance between two aircraft when resolving the conflict; 7 - states at different stages

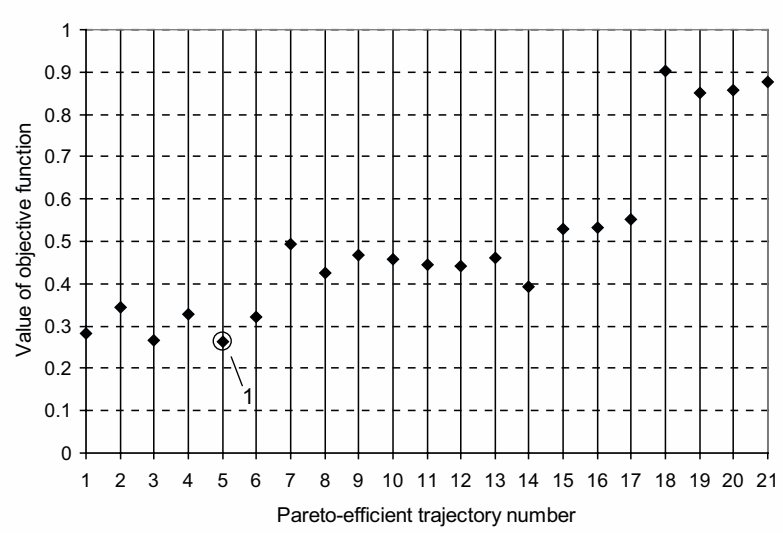

Fig. 3 . The value of the objective function, $\max _{\mathbf{W} \in \mathbf{D}_{w}} \sum_{i=1}^{3} w_{i} c_{i}(\mathbf{T}): 1-$
function minimum

Table 3. Specified flight speeds

\begin{tabular}{lccccccc}
\hline $\begin{array}{c}\text { Stage } \\
\text { number }\end{array}$ & 1 & 2 & 3 & 4 & 5 & 6 & 7 \\
\hline $\begin{array}{l}\text { Flight } \\
\text { speed }\end{array}$ & 220 & 220 & 225 & 230 & 225 & 220 & 220 \\
\hline
\end{tabular}

\section{Conclusions}

The method of multi-criteria synthesis of conflict-free flight trajectories has been described. This method includes the formation of a set of Pareto-effective conflict-free flight trajectories and is based on multi-criteria dynamic programming. The selection of optimal trajectories from the Pareto-efficient trajectories is carried out using the convolution of the vector optimality criterion.

The developed method, in contrast to the known methods, is based on the principle of multi-criteria optimization, when such separate criteria of optimality as the time of conflict resolution, fuel consumption, and the complexity of maneuvering, are used in combination. The synthesis of a conflict-free optimal trajectory can be performed by taking into account the real laws of the on-board flight management system using aircraft flight performances. This makes it possible to realize the calculated conflict-free trajectory by forming corresponding control signals to change the flight speed and heading separately or simultaneously. The use of dynamic programming for the sequential synthesis of conflict-free flight trajectories, enhances the computational efficiency.

The results of computer modeling prove the possibility of implementing the proposed conflict resolution method.

The synthesized conflict-free trajectory can be converted into a real ATC procedure for conflict resolution using area navigation (RNAV). The proposed method can be implemented when developing new ATC technologies and assessing the effectiveness of existing optimization methods of aircraft conflict resolution. 


\section{References}

Babak, V.; Kharchenko, V.; Vasylyev, V. 2007. Using generalized stochastic method to evaluate probability of conflict in controlled air traffic, Aviation 11(2): 31-36.

Bicchi, A.; Pallottino, L. 2000. On optimal cooperative conflict resolution for air traffic management systems, IEEE Transactions on Intelligent Transportation Systems 1(4): 221-232. https://doi.org/10.1109/6979.898228

Cafieri, S.; Brisset, P.; Durand, N. 2010. A mixed-integer optimization model for air traffic deconfliction, in Proceedings of the Toulouse Global Optimization Workshop TOGO 2010, August 2010, Toulouse, France, 27-30.

Cetek, C. 2009. Realistic speed change maneuvers for air traffic conflict avoidance and their impact on aircraft economics, International Journal of Civil Aviation 1(1): 62-73.

Eby, M. S. 1994. A self-organizational approach for resolving air traffic conflicts, The Lincoln Laboratory Journal 7(2): 239-254

Eby, M. S.; Kelly, W. E. 1999. Free Flight Separation Assurance Using Distributed Algorithms, in Proceedings of 1999 IEEE Aerospace Conference, 7 March 1999, Snowmass at Aspen, CO, USA, 2: 429-441. https://doi.org/10.1109/AERO.1999.793186

EUROCONTROL. 2003. CORA 2 Resolver Final report. Edition 1.0, Released Issue, NLR-CR-2003-458.

Frazzoli, E.; Mao, Z. H.; Oh, J. H., et al. 2001. Resolution of conflicts involving many aircraft via semi-definite programming, Journal of Guidance, Control, and Dynamics 24(1): 79-86. https://doi.org/10.2514/2.4678

Hu, J.; Prandini, M.; Sastry, S. 2000. Optimal maneuver for multiple aircraft conflict resolution: a braid point of view, in Proceedings of the 39th IEEE Conference on Decision and Control, 12-15 December 2000, Sydney, NSW, Australia, 4: 4164-4169. https://doi.org/10.1109/CDC.2000.912369
Kirwan, B.; Flynn, M. 2001. Identification of air traffic controller conflict resolution strategies for the CORA (Conflict Resolution Assistant) project, in Proceedings of the 4th USA/Europe Air Traffic Management R\&D Seminar, 3-7 December 2001, Santa Fe, NM, USA.

Klamroth, K.; Wiecek, M. M. 2000. Dynamic programming approaches to the multiple criteria knapsack problem, Naval Research Logistics 47(1): 57-76. https://doi. org/10.1002/(SICI) 1520-6750(200002)47:1<57::AIDNAV4>3.0.CO;2-4

Kosecka, J.; Tomlin, C.; Pappas, G., et al. 1997. Generation of conflict resolution maneuvers for air traffic management, in Proceedings of the 1997 IEEE/RSJ International Conference on Intelligent Robot and Systems 11-17 September 1997, IEEE, 3: 1598-1603.

Richards, A.; How, J. P. 2002. Aircraft trajectory planning with collision avoidance using mixed integer linear programming, in Proceedings of the 2002 American Control Conference 8-10 May 2002, Anchorage, AK, USA, 3: 1936-1941. https://doi.org/10.1109/ACC.2002.1023918

Tomlin, C; Pappas, G. J.; Sastry, S. 1998. Conflict resolution for air traffic management: a study in multiagent hybrid systems, in IEEE Transactions on Automatic Control 43(4): 509-521. https://doi.org/10.1109/9.664154

Vasyliev, D. 2014. Multi-objective synthesis of conflict-free aircraft flight trajectories, Science-based Technologies Journal 1(21): 37-40.

Zeghal, K. 1998. A review of different approaches based on force fields for airborne conflict resolution, in Proceedings of AIAA Guidance, Navigation, and Control Conference and Exhibit, 818-827. https://doi.org/10.2514/6.1998-4240 